\title{
Molecular Characterization and Functional Analysis of Wheat TtLOX Gene Involved in Aphid Resistance
}

\author{
Xinlun Liu ${ }^{1,2,3}$, Xiaolong Ma ${ }^{1}$, Xudan Kou ${ }^{1}$, Jinfeng Bai ${ }^{1}$, Hong Zhang ${ }^{1,2,3}$, \\ Changyou Wang $1,2,3$, Yajuan Wang ${ }^{1,2,3}$, Jixin Zhao ${ }^{1,2,3}$, Zengrong Tian ${ }^{1,2,3}$ \\ and Wanquan $\mathrm{Ji}^{1,2,3, *(\mathbb{B})}$ \\ 1 College of Agronomy, Northwest A\&F University, Yangling 712100, China; liuxinlun@nwafu.edu.cn (X.L.); \\ maxiaolong940803@163.com (X.M.); 2013015441@nwsuaf.edu.cn (X.K.); bjf199411@126.com (J.B.); \\ zhangh1129@nwafu.edu.cn (H.Z.); chywang2004@nwafu.edu.cn (C.W.); wangyj7604@nwafu.edu.cn (Y.W.); \\ zhaojixin@nwafu.edu.cn (J.Z.); tian.zr@163.com (Z.T.) \\ 2 State Key Laboratory of Crop Stress Biology for Arid Areas, Yangling 712100, China \\ 3 Shaanxi Research Station of Crop Gene Resources and Germplasm Enhancement, Ministry of Agriculture, \\ Yangling 712100, China \\ * Correspondence: jiwq1319@nwafu.edu.cn
}

Received: 26 April 2020; Accepted: 28 May 2020; Published: 30 May 2020

\begin{abstract}
The English grain aphid (Sitobion avenae F.) is one of the most important and destructive pests in wheat production worldwide. Lipoxygenase (LOX) is a key rate-limiting enzyme in the bio-synthesis pathway of jasmonate and methyl jasmonate in plants and animals. This enzyme is essential for plant growth and for biotic and abiotic stresses response. In this study, the TtLOX gene from wheat (Triticum turgidum) was isolated and characterized, and its conserved domains, phylogenetic relationship and subcellular localization were investigated. As a 2787-bp open reading frame (ORF) gene, TtLOX was predicted to encode a protein of 928-amino acid. TtLOX transcription was up-regulated in response to aphid feeding; the expression of TtLOX in wheat leaves was significantly higher in Lanmai, an aphid-resistant variety, than in Poland 305, an aphid-sensitive variety. In contrast, $T t L O X$ expression was down-regulated both in individuals treated with methyl-jasmine (MeJA) and in individuals with the TtLOX gene silenced by barley stripe mosaic virus (BSMV). The aphid numbers on the MeJA treated plants and on the TtLOX gene-silenced plants were higher than that on the control plants. These results suggest that the TtLOX gene may have potential as a target gene for controlling Sitobion avenae in wheat.
\end{abstract}

Keywords: wheat; Sitobion avenae; lipoxygenase (LOX); jasmonic acid synthesis; BSMV-VIGS

\section{Introduction}

Aphids are serious agricultural pests, which are a large group of insects of the Subfamily Aphidae feeding on phloem [1,2]. The English grain aphid (Sitobion avenae, EGA) is the dominant species of wheat aphids in Northern wheat region and Huang-Huai wheat region of China and other grain-growing areas worldwide [3,4], and causes significant yield losses in cereals throughout the world by phloem sap sucking [2], excreting honeydew [5] and transmitting plant viruses [6]. Chemical pesticides have been used to control aphids in wheat production world-wide, with undesirable environmental effects and an increase in insecticide resistance [7]. Thus, searching for natural plant resistance against aphids is of great significance [8]. The aphids have short life cycle with high fecundity, but their biological parameters can be affected by wheat varieties with different resistance traits [9]. In our study, we found most wheat cultivars were susceptible to aphid attack although aphid resistant genes were present in tetraploid wheat cultivars [10]. 
During the long evolutionary process, plants have evolved a variety of adaptations to reduce the damage caused by phytophagous insects. For example, an infestation of aphids elicits plant defense responses, which include toxic compounds [11] and structural defenses such as wax or trichomes on plant surfaces [12], callose [13], and accumulation of secondary metabolites [14]. In recent years, research focused on mechanisms of plant defense against aphids revealed that plants respond to aphids feeding through hormone signals. The plant hormones jasmonic acid (JA), salicylic acid (SA), abscisic acid (ABA) and ethylene were all shown to participate in aphid-induced resistance in plants $[15,16]$. The defense function of JA against phytophagous insects has been described in Arabidopsis [17] and other vascular plant such as barley [18], maize [19], wheat [16], soybean [20] and groundnut [21]. The importance of plant defense responses induced by exogenous applicating of JA or methyl jasmonate (MeJA) have been verified $[18,21]$. Lipoxygenases (LOXs) are a class of non-heme, iron-containing dioxygenases. LOXs, involved in encoding a key enzyme in the JA biosynthetic pathway, catalyzed the synthesis of traumatin, JA, MeJA and other oxylipins [22].

Plant LOXs are divided into two categories according to their position specificity and catalytic effect on hydrocarbon skeleton, namely 9-LOXs and 13-LOXs [22]. In plants, the products of LOXs may have a repellent effect on insect feeding. This repellency is the basis of insect resistance. LOXs may function as plant defense proteins, affecting aphid growth and development $[18,23]$. LOXs are required in aphid-infested plants to enhance the expression of defense regulatory gene. In cotton seedlings, for instance, LOXs activity were greatly induced by cotton aphid (Aphis gossypii) infestation [24]. In Arabidopsis, transcript levels of LOX2 and LOX5 increased after green peach aphid (Myzus persicae) (GPA) infestation [17,25]. In comparison to the wild-type (WT) plant, GPA spent less time feeding and GPA population was smaller on the Arabidopsis LOX5 mutant [25]. In rice, OsLOX1 is involved in resistance to brown planthopper $(\mathrm{BPH})$, transcripts accumulated rapidly and transiently in response to $\mathrm{BPH}$ attack, reaching a peak $6 \mathrm{~h}$ after initial feeding [23]. In barley, LOX2 was highly up-regulated after bird cherry-oat aphid (BCA) and GPA infestation [18,26,27]. LOX2 was found specifically induced by $\mathrm{BCA}$ feeding on the BCA resistant genotypes but not on the susceptible ones; the strength of up-regulation is positively correlated with the degree of resistance of the genotypes [27]. The fecundity of BCA and GPA was significantly lower on LOX2.2 overexpressing barley plants than that on the control plants, and the aphid tendency on anti LOX2.2 plants was also higher than that on the control plants [18].

In wheat, LOX1 was upregulated greatly in a wheat landrace carrying the Russian wheat aphid (Diuraphis noxia) (RWA) resistance gene Dnx, after RWA feeding [28]. Six transcripts similar to LOX genes were upregulated in resistant wheat plants after the greenbug aphid, Schizaphis graminum (Rondani) (GBA) feeding [16]. Our previous work showed that T. turgidum Lanmai, wheat landrace from China, was highly resistant to EGA [10]. A putative candidate EGA resistance gene (JK716074.1) of Lanmai, belonging to lipoxygenase gene family (LOX2), was found up-regulated in incompatible interaction through real-time PCR, following EGA infestation [29].

JA accumulates rapidly in response to injury or herbivore attack, inducing direct defensive capability at the site of attack and signaling transduction of the system's defense. As the first step of the key protein-catalyzed JA pathway, the LOXs are vital for understanding the defense mechanism of plants under ecological stress. Although the importance of LOXs is self-evident, there are few studies on the cloning of this defense gene in wheat.

In the present investigation, a 13-lipoxygenase gene, designated as TtLOX, was cloned and functionally characterized. We found that $T t L O X$ transcripts accumulated in wheat leaves after EGA feeding. Silencing of TtLOX and exogenously applied methyl jasmine suppressed wheat resistance and reduced aphids' fecundity while suppressing the expression of TtLOX. Our data indicate that TtLOX is involved in wheat against aphid and the regulation of jasmonate biosynthesis. 


\section{Materials and Methods}

\subsection{Plant Cultivation and Aphid Rearing}

Three tetraploid wheat varieties that differed in EGA resistance levels were used. Variety Lanmai (Shaanxi, Zhashui)(T. turgidum) was highly resistance to EGA, variety Poland 305 (T. polonicum) was moderately susceptible to EGA and variety and variety Xiaoyan 6 (T. aestivum) was highly susceptible to EGA [10]. Seeds of the three varieties and tobacco cultivar Nicotiana benthamiana (Nicotiana tabacum) were obtained from Northwest A\&F University.

All seeds were germinated in a $23{ }^{\circ} \mathrm{C}$ incubator and were sown in plastic pots filled with planting soil (containing peat and vermiculite at a 3:1 ratio) in a growth chamber. The wheat plants were grown under standard conditions at $21 \pm 1{ }^{\circ} \mathrm{C}$, and a $16 \mathrm{~h}$ photoperiod at $150 \mu \mathrm{mol}$ photons $\mathrm{m}^{-2} \mathrm{~s}^{-1}$, relative humidity $50 \%-60 \%$. The tobacco plants were grown under $25^{\circ} \mathrm{C}$ and a $16 \mathrm{~h}$ photoperiod at $150 \mu \mathrm{mol}$ photons $\mathrm{m}^{-2} \mathrm{~s}^{-1}$, relative humidity $65 \%-70 \%$.

A single isolate aphid of EGA was collected in a wheat field in Yangling, Shaanxi Province, China, and has been reared on wheat cultivar Xiaoyan 6 in a growth chamber with conditions as described above.

\subsection{Experimental Procedure}

The expression of TtLOX was measured in leaves of Lanmai and Poland 305 at two-leaf and one-heart stage under the stress of EGA. The experiments were conducted in a growth chamber, consisting of three replicates (each replicate contains two groups, of 30 seedlings each). Each individual plant was inoculated with two same-sized adult EGAs and were placed within a plastic housing to prevent the escaping of EGAs [30]. The culture conditions were the same as the aphids' culture. Samples (about $1 \mathrm{~cm}^{2}$ each) were collected from 3 plants of each genotype in each replicate at $0,12,24$, 48,72 and $96 \mathrm{~h}$ after inoculation; all samples were immediately frozen in liquid nitrogen and stored at $-80^{\circ} \mathrm{C}$ fridge until further testing.

The effects of MeJA on the expression of TtLOX were studied in seedlings of Lanmai at two-leaf and one-heart stage. The plants were treated with $1 \mathrm{mmol} / \mathrm{L}$ methyl jasmonate [31], and the plants of similar size were sprayed with the solution for about $30 \mathrm{~s}$ until the surface of the leaves began to drip, a protective shield was then put around the plant to keep it from evaporating [32]. The leaves were harvested at 0,12, 24, 48 and $72 \mathrm{~h}$ after treatment. At $72 \mathrm{~h}$ after treatment, each plant was inoculated with 5 same-sized adult EGAs, and a protective shield was put again around the treated plant. The plants were then cultured under the same conditions as that if the aphid culture. The number of aphids per plant was counted at 7, 14 and 21 days. MeJA (Purity $>95 \%$ ) was purchased from Solarbio (Beijing, China). The MeJA was first dissolved in small quantity of absolute alcohol and then diluted. Distilled water was used as control. All samples were immediately frozen in liquid nitrogen and stored at $-80^{\circ} \mathrm{C}$ fridge until used for RNA extraction.

In order to study the effect of TtLOX silencing on wheat resistance to EGA at the seedling stage, the barley stripe mosaic virus (BSMV) mixed RNA $(\alpha, \beta, \gamma / \gamma$-PDS $/ \gamma$-TtLOX) transcribed in vitro was rubbed evenly on the leaves of two-leaf and single-leaf stage (until the second leaf is completely flattened) [33], the leaves were harvested 15 days after treatment and were frozen in liquid nitrogen and stored at $-80^{\circ} \mathrm{C}$ fridge until expression analysis. Distilled water was used as control. Aphid inoculation was performed 20 days after virus inoculation; the method of aphid inoculation was the same as that of the MeJA treatment experiment. The number of aphids per plant was counted at day 7 and 14 after inoculation.

\subsection{Cloning and Bioinformatics Analyses of TtLOX}

Total RNA of Lanmai leaves was extracted using RNAiso Plus (Takara Biotechnology, Beijing, China). The mass and concentration of RNA were determined by $1 \%$ agarose gel electrophoresis and 
spectrometric analysis. The total RNA was reverse transcribed into cDNA template using Takara PrimeScript $^{\mathrm{TM}}$ 1st strand cDNA synthesis kit (TaKaRa Biotechnology, Beijing, China).

The EST sequence in mRNA differential display library was BLAST using GrainGenes website (https://wheat.pw.usda.gov/cgi-bin/seqserve/blast_wheat.cgi) to get the highest homology sequence. ORF analysis was performed with ORF finder (https://www.ncbi.nlm.nih.gov/orffinder). The specific primers TtLOX-f and TtLOX-r (Table 1) were designed and the CDS of TtLOX was cloned. The PCR products were detected, purified and cloned into $\mathrm{pMD}^{\mathrm{TM}} 19-\mathrm{T}$ Vector (TaKaRa), and then transformated into E. Coli DH5 $\alpha$. Monoclonal colonies were selected ampicillin resistance $\left(50 \mu \mathrm{g} \mathrm{ml}^{-1}\right)$ and screened for TtLOX insert by PCR. The transformants selected were transferred into LB broth and cultivated at $37^{\circ} \mathrm{C}$, and then sequenced (AuGCT Biotechnology). The full-length sequence of TtLOX was cloned by using PrimeSTAR ${ }^{\circledR}$ HS DNA Polymerase (TaKaRa) after the cDNA was diluted to the appropriate concentration by EasyDillution (TaKaRa). The cloning PCR protocol included pre-denaturation at $98^{\circ} \mathrm{C}$ for $2 \mathrm{~min}$ followed by 35 cycles at $98^{\circ} \mathrm{C}$ for $15 \mathrm{~s}, 58^{\circ} \mathrm{C}$ for $5 \mathrm{~s}$ and $72{ }^{\circ} \mathrm{C}$ for $3 \mathrm{~min}$, and $72{ }^{\circ} \mathrm{C}$ for $5 \mathrm{~min}$.

Table 1. Primers used in this study.

\begin{tabular}{cc}
\hline Primer Name & ${\text { Primer Sequence } \mathbf{5}^{\prime} \rightarrow \mathbf{3}^{\prime}}^{\text {Tt } L O X-\mathrm{f}}$ CTATAAATAGGATCCAGGGC \\
Tt $L O X-\mathrm{r}$ & CCTTTTATAGCATTCCGT \\
GFP-TtLOX-f & TAGCCATGGTAGATCTGATGCTAACGGCGACGAAGTC \\
GFP-TtLOX-r & ACAGGCCTTACGTATCAAATGGAGATGCTGTTGG \\
VIGS-TtLOX-f & ACGGGCATAAAGACCGCCAA \\
VIGS-TtLOX-r & CTTGAGGACGTATGGCAGCA \\
VIGS-PDS-f & TTCACTGTTCCGTCCGGGTT \\
VIGS-PDS-r & AAGCAGGGTGTCCTGATCG \\
qPCR-TtLOX-f & AAGTTGGACGAGGCAACCTAC \\
qPCR-TtLOX-r & AGAGCTTCTTGTTAGCCACGG \\
qPCR-PDS-f & TTCACTGTTCCGTCAGGGTTC \\
qPCR-PDS-r & CAGTCTTTGGGTGGTGAGGTC \\
qPCR-BSMV-f & AACTGCCAATCGTGAGTAGGTT \\
qPCR-BSMV-r & CTCCTGTTCAGAACGTTTCAGAAGT \\
Actin-f & TAGGAGGGCAAGTCTGGT \\
Actin-r & CTTTCGCAGTTGTTCGTC \\
\hline
\end{tabular}

The chromosome map was produced using MapGene2Chrom web v2 (http://mg2c.iask.in/mg2c v2.0). The analyse of the conserved domain of TtLOX was conducted using the conservative domain search tool Pfam (http://pfam.xfam.org/search\#tabview=tab0). The physical and chemical properties of TtLOX was analyzed using Protparam (https://web.expasy.org/protparam).. The subcellular localization prediction was performed using the PSORT tool on the GENSCRIPT website (https://www.genscript. com/psort.html). TtLOX motif analysis was conducted using MEME (http://meme-suite.org/tools/ meme). Sequence multiple alignment was determined using DNAMAN 8.0 (Lynnon Biosoft of Quebec, Canada). The phylogenetic analysis was carried out using the adjacent joining method of Mega 7 (Biodesign Institute, Tempe, AZ, USA). The confidence of each branch was evaluated using 1000 repeated bootstrap analysis. The 2000bp upstream of the start codon (ATG) of TtLOX for T. turgidum genome sequence was carried out using the promoter analysis; the possible cis-regulatory elements in the sequence was analyzed using the PlantCARE database (Vlaams Instituut voor Biotechnologie).

\subsection{Subcellular Localization of TtLOX Fusion Protein in Tobacco}

The specific primers (GFP-TtLOX-f and GFP-TtLOX-r) containing homologous arms were designed with the full-length coding sequence of TtLOX (Table 1). The amplified target fragment and plant expression vector $p C H F 3-e G F P$ were recombined to construct $p C H F 3-e G F P$ :TtLOX using ClonExpress II One Step Cloning Kit (Vazyme Biotech, Nanjing, China). pCHF3-eGFP was used as the control. 
The construct $p C H F 3-e G F P: T t L O X$ and $p C H F 3-e G F P$ plasmids were separately transformed into Agrobacterium tumefaciens EHA105, and then were infiltrated into N. benthamiana leaves. The main process is as following: A. tumefaciens containing transformed plasmids and empty plasmids were cultured to a suitable concentration, and the $A$.tumefaciens was mixed with the permeate solution (250 mg D-glucose, $5 \mathrm{~mL}$ MES solution, $5 \mathrm{~mL} \mathrm{MgCl}_{2}$ solution, $5 \mu$ acetyl eugenone solution, ddH2O up to $50 \mathrm{~mL}$ ). After the waxy layer of $N$. benthamiana leaves surface was gently removed with syringe needle, the mixture spread through the leaf by slowly pushing the syringe until water drips out. The infected tobacco plants were marked and the transformed plants were kept out of light until being observed. After being incubated for $48 \mathrm{~h}$ at $25^{\circ} \mathrm{C}$, the infected leaves were cut and placed on the slide, then sterile water was added, and the slide was covered and pressed tightly. The leaves were then assessed using fluorescence confocal microscope (Bx52+DP72, Olympus, Japan).

\subsection{Gene Silencing Based on BSMV in T. turgidum}

Gene silencing for T. turgidum was carried out using the same gene silencing system of common wheat [34]. Specific primers of the 497-bp target fragment were designed according to the coding sequence of TtLOX (Table 1). Phenotypic marker gene phytoene desaturase 3 (TRIDC4BG051300.4, PDS) was obtained from Graingenes (https://wheat.pw.usda.gov/cgi-bin/seqserve/blast_link_result. cgi\#TRIDC4BG051300.4) and specific primers were designed (Table 1) to amplify the 277-bp PDS fragment. The TtLOX and PDS fragments were linked to BSMV vector to form $\gamma$-TtLOX and $\gamma-P D S$ fusion vector, respectively. The $\gamma$-PDS vector was the phenotypic marker and $\gamma$ was the control. The main process is as following: the recombinant plasmids $\alpha, \beta, \gamma, \gamma-P D S, \gamma-T t L O X$ were linearized by using $\mathrm{X} \mathrm{cmI}$ (New England Biolabs) and linked to the $\gamma$-PCR vector, respectively. The plasmids were used as restriction enzyme: $\alpha, \beta$ : Mlu I; $\beta$ : Spe I; $\gamma$-PDS and $\gamma$-TtLOX: BssH II. The linearized plasmid was transcribed in vitro using RiboMAX ${ }^{\mathrm{TM}}$ Large Scale RNA Production System (Promega). The in vitro transcripts of $\alpha, \beta, \gamma, \gamma$-PDS and $\gamma$-TtLOX target genes were mixed $10 \mu l$ each according to $\alpha, \beta$ and $\gamma / \gamma-P D S / \gamma-T t L O X$, and $30 \mu \mathrm{l}$ plasmid mixed solution was obtained from each treatment. The final permeate was prepared by adding $220 \mu \mathrm{l} \mathrm{GKP} \mathrm{permeate} \mathrm{(} 50 \mathrm{mM} \mathrm{Gly,} 30 \mathrm{mM} \mathrm{K} \mathrm{HPO}_{4}, 1 \%$ bentonite, $1 \%$ diatomite). Then, $22 \mu \mathrm{l}$ inoculant was dripped to the finger of sterile gloves and the two fingers were kneaded together to make the liquid stick evenly. Then the second leaf was gently rubbed from the base to the tip. This was repeated $3 \times$ until the inoculant was completely absorbed by the leaf.

\section{6. qRT-PCR Analysis}

Total RNA was extracted from the expanded leaves under aphid stress, MeJA treatment and VIGS experiment, using RNAiso Plus (Takara), then reverse-transcribed into cDNA using PrimeScript ${ }^{\mathrm{TM}} \mathrm{RT}$ reagent Kit (TaKaRa). The expression levels of TtLOX, PDS and BSMV were determined by quantitative real-time PCR using SYBR Premix Ex Taq (Takara). RT-qPCR was performed using Applied Biosystems Q7 Real-time PCR system (Thermo Fisher Scientific).

The specific primers used for TtLOX (qPCR-TtLOX-f and qPCR-TtLOX-r) expression analysis, PDS (qPCR-PDS-f and qPCR-PDS-r) expression analysis and BSMV accumulation detection (qPCR-BSMV-f and qPCR-BSMV-r) are shown in Table $1 . \beta$-actin is used as the internal reference gene. The real-time RT-PCR conditions were as follows: $2 \mathrm{~min}$ at $95^{\circ} \mathrm{C}$ for denaturation, followed by 45 cycles of $8 \mathrm{~s}$ at $95^{\circ} \mathrm{C}, 30 \mathrm{~s}$ at $58^{\circ} \mathrm{C}$, and $20 \mathrm{~s}$ at $72{ }^{\circ} \mathrm{C}$ for amplification. Each analysis comprised of three technical replicates and three biological replicates. The gene expression levels were quantified using the 2- $\Delta \Delta \mathrm{Ct}$ method [35]. Graphpad Prism 8 (San Diego, California) was used for drawing.

\subsection{Statistical Analysis}

Data analysis was performed using SPSS 25.0 software. The data from the quantitative TtLOX assay were subjected to analysis of variance and the mean values were compared using the LSD test $(p=0.05)$. Data were compared using two-way ANOVA, ${ }^{*}(p<0.05)$ and ${ }^{* *}(p<0.01)$ designate that the differences are statistically significant and highly significant, respectively. 


\section{Results}

\subsection{Isolation and Characteristic Analysis of the TtLOX cDNA Sequence}

The EST sequence (JK716074.1), from the SSH-cDNA library of Lanmai seedling leaves induced by EGA [29], was compared to lipoxygenase gene in the Triticum dicoccoides (TRIDC5AG001270.2, 92\%). Using specific primers TtLOX-f and TtLOX-r amplified the full-length coding sequence of TtLOX and the UTR region of 5'-Terminal $185 \mathrm{bp}$ and 3'-Terminal $94 \mathrm{bp}$. The 2,787-bp TtLOX ORF sequence isolated from Lanmai encoding a polypeptide of 928-amino acid, the molecular weight was $105.45 \mathrm{kDA}$ and the isoelectric point was 5.73. The full-length sequence of TtLOX was in full agreement $(100 \%)$ with the predicted transcript TRIDC5AG001270.2 in the T. dicoccoides genome. The sequence was also highly homologous with Triticum turgidum and Triticum aestivum LOX gene sequence, with 99.9\% similarity to the predicted transcripts TRITD5Av1G002820 in durum wheat and $99.6 \%$ similarity to the common wheat TraesCS5A02G007900. The TtLOX gene fragment was located at 4,967,647-4,970,556 of the 5A chromosome, containing 5 exons and 4 introns (Supplementary Materials Figure S1), the exons and introns are represented by rectangles and polylines respectively). Protein conserved domain analysis revealed that TtLOX had three conserved LOX domains (Supplementary Materials Figure S2), which are considered as binding, catalytic and regulatory sites for LOX, with a similarity ranging from $99.78 \%$ (TuLOX) to $69.45 \%$ (HvLOX). TtLOX was therefore designated as a member of the LOX family. To confirm the homology between TtLOX and the LOX family, a phylogenetic tree was constructed using one LOX from Mus musculus, 24 LOXs from other plant species and TtLOX. The phylogenetic tree was divided into 9-LOX and 13-LOX by difference in number of branches. TtLOX belongs to 13-LOX, and is highly similar to TuLOX (Supplementary Materials Figure S3). Analysis of the sequence immediately upstream of the transcription start site indicated that the promoter region of TtLOX gene contained putative binding sites for hormonal response related cis-regulatory element of transcription ABA, auxin, MeJA, GA, SA, defense and stress responsiveness (Table 2).

Table 2. Putative binding sites for the activators/regulators of hormonal response.

\begin{tabular}{cc}
\hline Function & Cis-Regulatory Element \\
\hline ABRE & Abscisic acid (ABA) responsiveness \\
AuxRR-core & Auxin responsiveness \\
CGTCA-motifTGACG-motif & MeJA-responsiveness \\
GARE-motif & Gibberellin (GA) -responsive \\
TC-rich repeats & Defense and stress responsiveness \\
TCA-element & Salicylic acid (SA) responsiveness \\
\hline
\end{tabular}

\subsection{Subcellular Localization}

The subcellular localization of a protein provides valuable information about its possible functions. Therefore, the $p$ CHF3-eGFP:TtLOX and $p$ CHF3-eGFP constructs were separately transiently introduced into tobacco leaves to examine the subcellular localization of $T t L O X$. The fluorescence signal of $p C H F 3-e G F P$ was evenly distributed in the nucleus and cytoplasm, but the fluorescent signal of pCHF3-eGFP:TtLOX was concentrated on the cell membrane and nucleus (Figure 1). These findings were consistent with our bioinformatics prediction.

\subsection{Expression of TtLOX in Tetraploid Wheat under EGA Stress}

Jasmonates are involved in defense against herbivores, and LOX is a key enzyme in jasmonate biosynthesis signaling pathway. To determine the response of TtLOX to EGA, qRT-PCR was performed to investigate the transcript levels of TtLOX in aphid-resistant tetraploid wheat variety Lanmai and aphid-sensitive tetraploid wheat variety Poland 305 after EGA infestation (Figure 2). The results showed that TtLOX transcript level was upregulated within $12 \mathrm{~h}$ after EGA infestation. The expression level was higher in Lanmai than $\mathrm{t}$ in Poland 305 at all time points except $72 \mathrm{~h}$. After EGA infestation, 
the expression level of TtLOX in Poland 305 increased gradually and reached its highest value at $96 \mathrm{~h}$ with 10.5-fold the level of that at $0 \mathrm{~h}$. In comparison, the expression level of TtLOX in Lanmai increased rapidly after ECA infestation and reached its highest value at $48 \mathrm{~h}$ with 38.8 -fold the level of that at $0 \mathrm{~h}$. the expression level was then decreased to 7.5-fold and 15.1-fold (level of $0 \mathrm{~h}$ ) at $72 \mathrm{~h}$ and $96 \mathrm{~h}$, respectively.

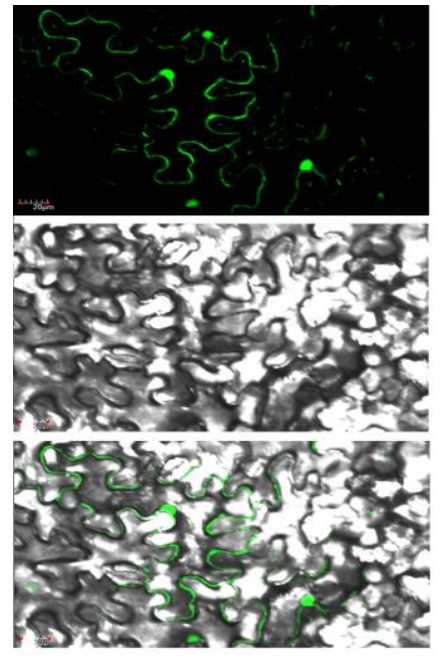

pCHF3-eGFP

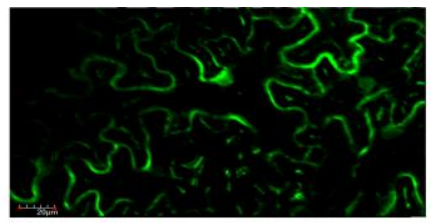

\section{Flourescence}

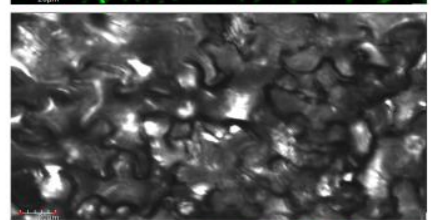

Bright field

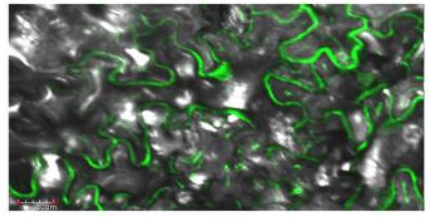

\section{Merge}

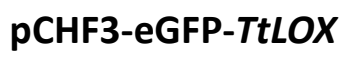

Figure 1. Subcellular localization of TtLOX in transiently expressing in epidermal cells of N. benthamiana leaf. Bars $=50 \mu \mathrm{m}$.

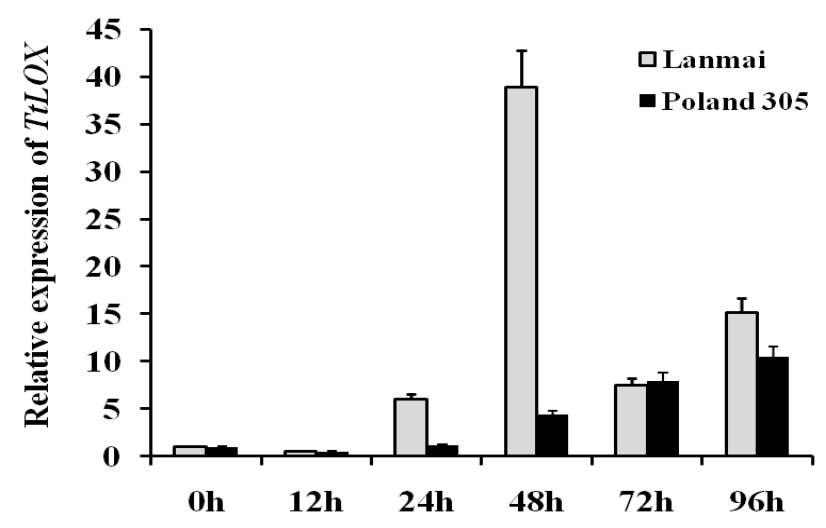

Figure 2. Relative expression levels of TtLOX in different tetraploid wheat genotypes under English grain aphid (EGA) stress.

\subsection{Changes in TtLOX Expression Levels and EGA Numbers under MeJA Treatment in T. turgidum}

In order to study whether TtLOX could indirectly protect wheat by regulating the synthesis of endogenous MeJA, MeJA was used to study whether the expression of TtLOX and the level of wheat resistance to aphids were affected. After MeJA treatment, the expression level of TtLOX in Lanmai decreased rapidly and was always lower than that of the mock treatment (Figure 3a). At $12 \mathrm{~h}$ and $24 \mathrm{~h}$, there was no significant difference in TtLOX transcription between the MeJA treatment group and mock treatment group. However, after $48 \mathrm{~h}$ and $72 \mathrm{~h}$ of MeJA treatment, TtLOX expression levels in the mock treatment plants were 20.8 times (two-way ANOVA: F1,5 =10.08, $p=0.01$ ) and 4.8 times (two-way ANOVA: $F 1,5=2.12, p=0.18$ ) higher than those in the MeJA treatment plants, respectively. 


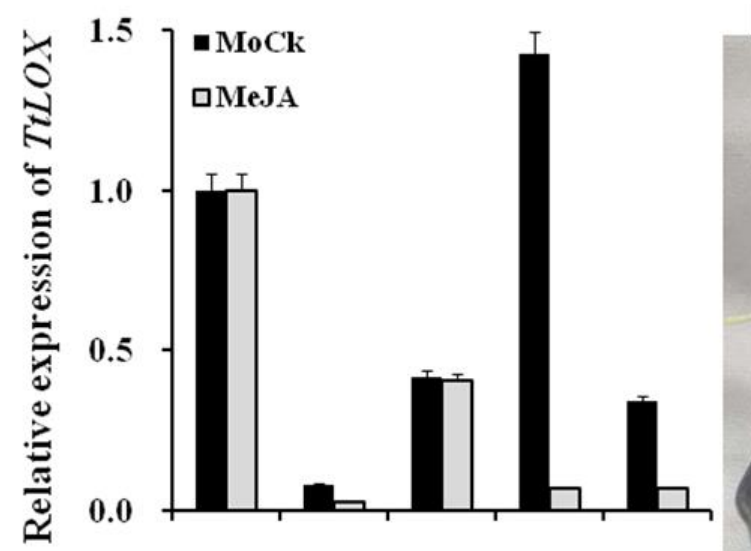

(a)

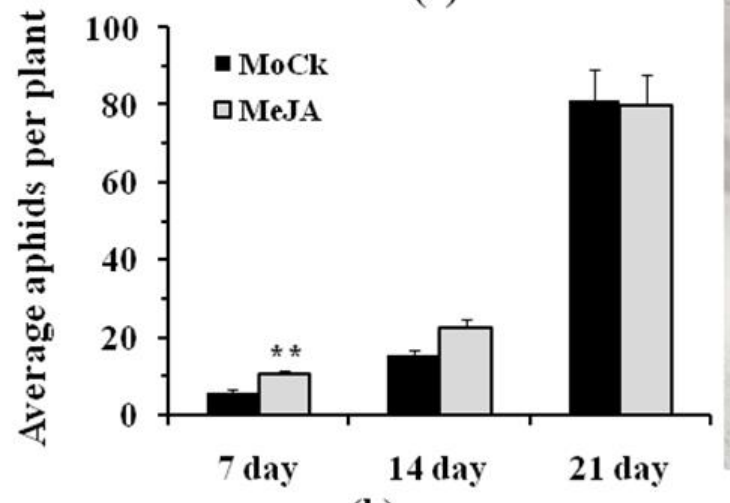

(b)

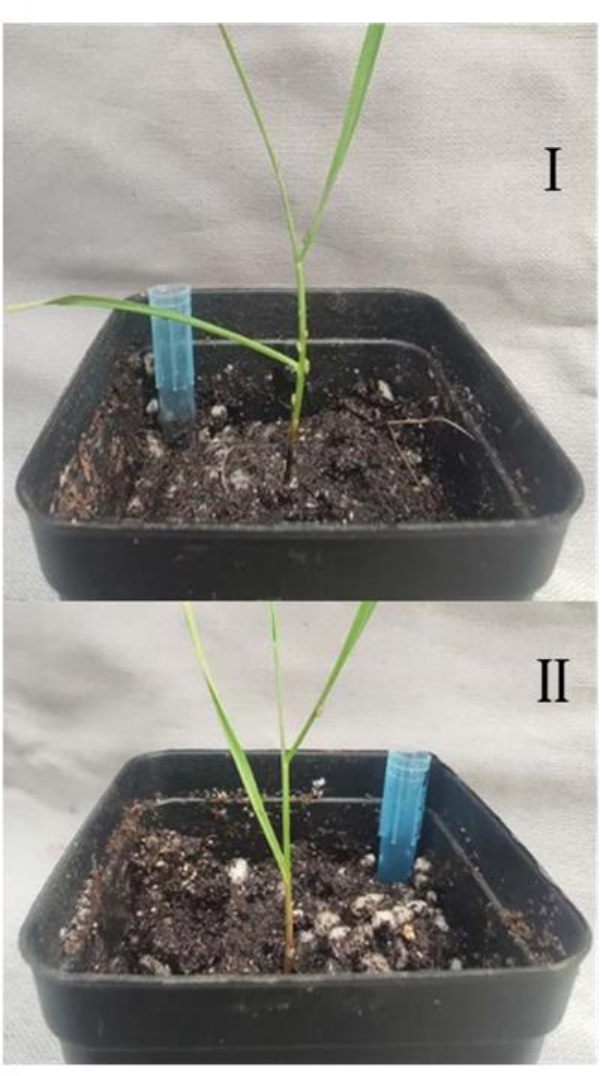

(c)

Figure 3. Expression and EGA resistance levels of TtLOX in wheat after MeJA treatment. (a) The change of TtLOX expression levels after MeJA treatment compared with mock treatment. Data are means $\pm \operatorname{SEM}(n=3)$. (b) Comparison of the average EGA number per plant between MeJA treatment group and mock treatment group $(n=5)$ at day 7,14 and 21 after aphid inoculation. Data are means \pm SEM $(n=9)$. (c) Plant of MeJA treatment (I) mock treatment (II). MeJA: methyl jasmonate. Asterisks indicates a significant difference between MeJA treatment plants and mock plants: ${ }^{*} p \leq 0.05$; ** $p \leq 0.01$.

To detect the possible effect of MeJA treatment on aphid fecundity, each Lanmai plant of both the MeJA treatment group and the mock treatment was inoculated with 5 synchronous apterous EGA, $72 \mathrm{~h}$ after MeJA treatment, and the number of aphids on each plant was counted after day 7, 14 and 21. The results showed that the number of aphids was significantly higher in the MeJA treatment group than in the mock treatment group (Figure 3b,c) at day 7 and 14, but not at day 21 .

\subsection{Gene Silencing and Changes in Aphid Reproduction}

Virus-induced gene silencing (VIGS) is an effective tool for studying gene function in plants. Barley stripe mosaic virus (BSMV)-VIGS is widely used in monocotyledonous plants. To evaluate the role of TtLOX in wheat resistance to EGA, we introduced a BSMV-VIGS construct of TtLOX into the wheat variety Lanmai. A total of $21 \mathrm{TtLOX}$-VIGS individual plants were acquired. We investigated the aphid reproduction and the transcript level of TtLOX in both TtLOX-silenced and non-silenced plants.

Two weeks after infection, BSMV accumulation on infected plants was more than 1000 times higher than that on the mock plants (Figure 4a,b). The expression levels of TtLOX and PDS on infected plants decreased to 30 percent and 59 percent of that on mock treated plants, respectively (Figure 4c,d). At day 30, the leaves of BSMV:PDS infected plants appeared mottled with photobleaching phenotype; at day 35, the phenomenon was more obvious (Figure 4f). In comparison, the untransformed plants did not have this phenotype, indicating this experiment was successful. 


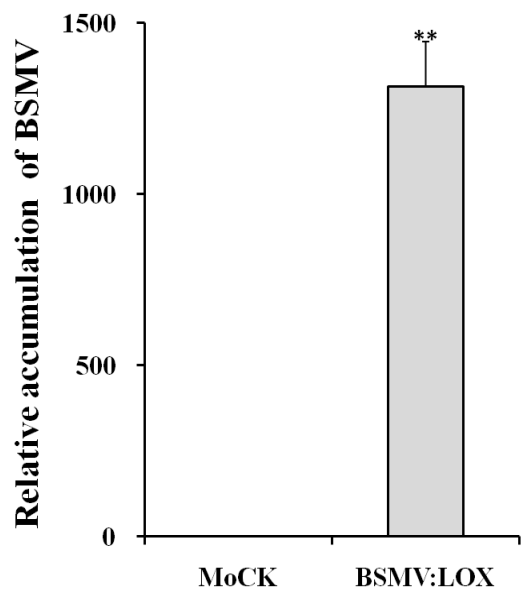

(a)

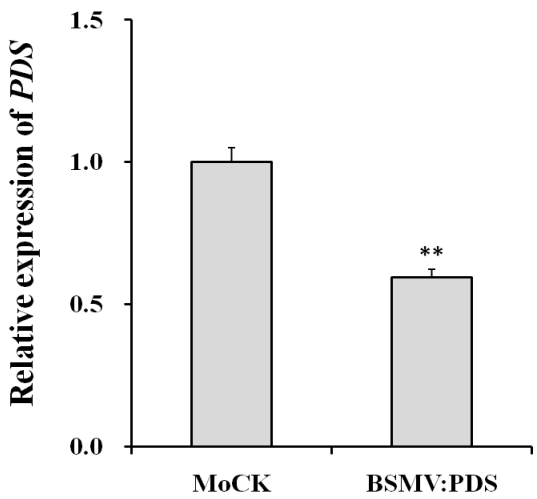

(c)

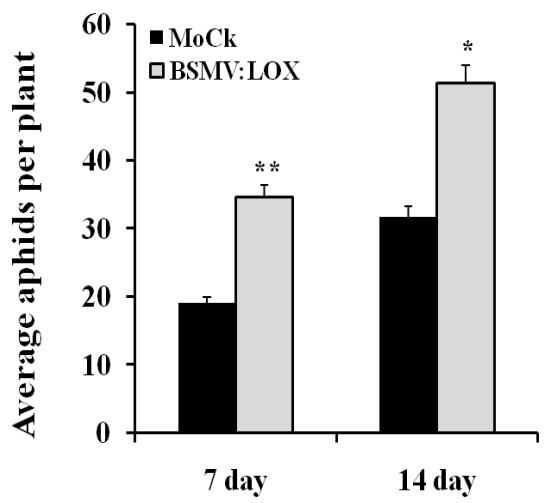

(e)

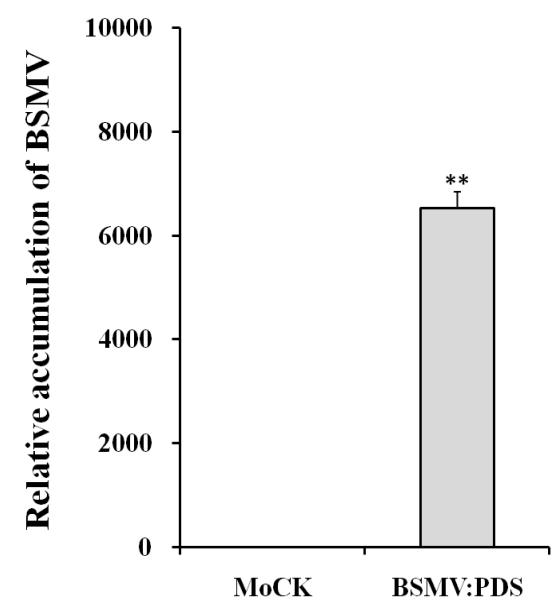

(b)

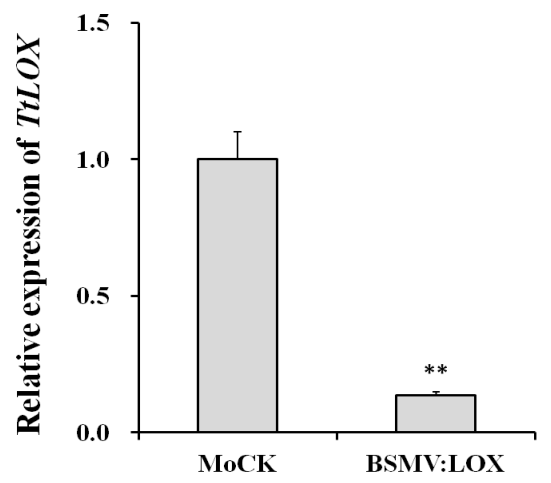

(d)

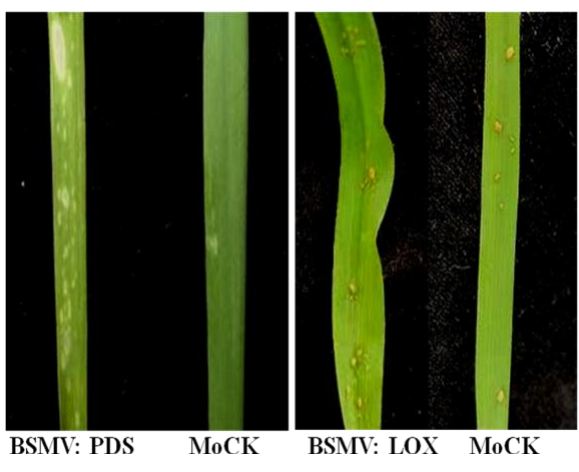

(g)

Figure 4. Functional validation of TtLOX with BSMV-VIGS. (a) and (b) are the BSMV accumulation levels in the BSMV:PDS and BSMV:LOX transformed Lanmai leaves 14 days after inoculation. (c) and (d) are the target genes expression levels in BSMV:PDS, BSMV:LOX and mock groups 14 days after virus inoculation. (e) Comparison of the average EGA number per plant between the mock group and BSMV:LOX treatment group at 7 and 14 days after aphid inoculation. (f) The albino phenomenon of the BSMV:PDS treated lanmai leaf at day 35. (g) The EGAs on wheat leaves of BSMV:LOX plants at day 7 after aphid inoculation. Data are means \pm SEM $(n=9)$. BSMV:PDS-virus infection was performed by inserting a PDS fragment; BSMV:LOX-virus infection was performed by inserting a LOX fragment. Asterisks indicate significant differences between BSMV:LOX/PDS treatment plants and mock plants $\left({ }^{*} p \leq 0.05 ;{ }^{* *} p \leq 0.01\right)$.

The plants of BSMV:TtLOX were inoculated with aphids after two weeks of virus infection, and the aphids number per plant were counted at day 7 and 14 after infection (Figure 4e). The results 
showed the number of aphids per BSMV:TtLOX treatment plant were significantly higher than that of the non-silenced plant, indicating the decrease of TtLOX expression level would lead to a decrease in aphids resistance in wheat (Figure 4e,g). This result is also consistent with our previous MeJA treatment experiments.

\section{Discussion}

Jasmonate acid signaling molecules can effectively mediate plant defense responses to insect [20,36]. Lipoxygenase, as a key factor of jasmonic acid synthesis, has been previously studied in several plant species in responses to aphids' infestation [18,23-25]. However, little is known about the lipoxygenase for wheat. The present study was conducted to clone TtLOX from T. turgidum and study the role of TtLOX against EGA in wheat.

Our results suggest that TtLOX might have a signal peptide according to PSG and GvH signal peptide, and the predicted protein was 928 amino acids with a PLAT/LH2 domain and a lipoxygenase superfamily domain (Supplementary Materials Figure S2). Multiple sequence analysis of TtLOX and other plant LOXs showed that two highly homologous domains existed in TtLOX, which is very important for the synthesis, catalysis and functional activity of LOXs [22,37]. Similarly, the protein contains five conserved amino acid residues that coordinate with water or hydroxide with non-heme-iron in the active center. The Ala and Phe locis of the putative protein suggest that the TtLOX belongs to the 13-LOX, consistent with our previous predictions and the regular pattern of jasmonic acid biosynthesis [38]. The phylogenetic tree analysis suggests that $T t L O X$ was a type 13-LOX (Supplementary Materials Figure S3). Moreover, OsLOX1, ZmLOX10 and the barley LOX2.2 are both characterized as 13-LOX, and are believed to be involved in resistance against the brown plant hopper, Spodoptera exigua and aphids, respectively $[18,19,23]$. Apart from their functions in JA biosynthesis, LOXs are vital for green leaf volatile (GLV) biosynthesis [19]. GLVs play key roles in plant defenses and are responsible for plant-insect interactions [19,39]. Several types of LOXs associated with insect resistance have been localized in chloroplasts accordingly [18,19,23,40,41]. However, TtLOX was localized to the nucleus and membrane, which was not consistent with previous studies. ZmLOX10 has been localized to non-chloroplast organelles [19]. Our results did not show conclusively about the involvement of GLVs signal transduction in wheat against EGA. Further research on this subject is warranted.

The expression levels of TtLOX in the aphid-resistant wheat variety Lanmai and the susceptible variety Poland 305 after aphids infestation were analyzed by real-time PCR. The results showed that the expression levels of TtLOX in Poland 305 were lower than that in Lanmai (Figure 2). The results are consistent with previous studies for other plant species such as tobacco infected by cucumber mosaic cucumovirus (CMV) [42], and peanutinfected by Aspergillus parasiticus [43]. After S. avenae feeding, the expression of TtLOX in Lanmai increased rapidly after 24 hpost infestion (hpi) and peaked at $48 \mathrm{hpi}$, but TtLOX response in Poland 305 was delayed which increased at $48 \mathrm{hpi}$ and peaked at 96 hpi (Figure 2). Similarly, after cotton aphid (Aphis gossypii) feeding, the specific activity of LOX in cotton seedlings increased by $18 \%$ at $24 \mathrm{~h}, 34 \%$ at $48 \mathrm{~h}$ and $24 \%$ at $72 \mathrm{~h}$, respectively [24]. After feeding the greenbug aphid Schizaphis graminum (Rondani), the expression of 6 transcripts similar to LOXs increased significantly in resistant wheat varieties at $24 \mathrm{hpi}$ and $48 \mathrm{hpi}$ [16]. After RWA feeding, the expression levels of several oxylipin-based defense signals genes in RWA-resistance wheat strains, containing the RWA resistance gene Dnx, increased significantly from 6- to 24-hpi, but the increasing of these genes were delayed to 48- to 96-hpi in RWA-susceptible wheat strains [28].

In this study, the TtLOX expression in MeJA treated plants was significantly lower than that in mock treated plants (Figure 3a). These results indicate that exogenous MeJA had negative feedback regulation on TtLOX gene expression, which explains the further reduction of aphid resistance in the plant. After MeJA induction, the aphids' fecundity was increased and sustained for at least 14 days (Figure 3b). This is consistent with the findings in tobacco [44]. Studies in cucumbers showed that different amounts of exogenous jasmonic acid treatment had different effects on endogenous jasmonic 
acid synthesis pathway [45], and the plant perception of jasmonic acid was similar to that of IAA [46]. Although most previous studies have shown that JAs have positive feedback control on jasmonic acid synthesis, there are also examples of negative feedback control. The expression level of LOX in avocado fruit 'Hass' treated with different concentrations of MeJA decreased, asthe MeJA concentration increased [47]. In response to MeJA treatment of the poplar resin oxygenase gene family, although most of the LOXs gene showed up-regulation or no change after MeJA treatment, PtLOX3 showed down-regulation after the treatment [48].

In the present study, the average number of aphids per TtLOX-silenced plant was significantly higher than that of the control despite 7-fold down-regulation of TtLOX (Figure 4e). The increase in the aphids fecundity of both the TtLOX-silenced plants and the MeJA treatment plants suggests that $T t L O X$ is positively correlated with aphid resistance. Thus, our results strongly suggest that TtLOX is inducible by aphid feeding and contributes to plant resistance to EGA feeding; TtLOX may regulate aphid feeding behavior and population growth by regulating jasmonic acid biosynthesis in T. turgidum.

Supplementary Materials: The following are available online at http://www.mdpi.com/2073-4395/10/6/780/s1, Figure S1: Gene Structure and chromosome location of TtLOX; Figure S2: Alignment of the putative TtLOX sequence with LOXs in other plants; Figure S3: Phylogenetic analysis of TtLOX in plants.

Author Contributions: X.L. and W.J. designed the study, analyzed the data and wrote the article; X.L. and X.M. performed the research; X.L., C.W. and Y.W. contributed to the development of material; X.L., X.M., X.K. and J.B. contributed to gene cloning, functional analysis and data analysis; H.Z., J.Z. and Z.T. contributed to aphid resistance. All authors have read and agreed to the published version of the manuscript.

Funding: This research was funded by the National Natural Science Foundation China (No. 31871614), the National Key Research and Development Program of China (No. 2016YFD0100102), Crop Germplasm Resources Protection (No. 2019NWB036-02-1), and the Programme of Introducing Talents of Innovative Discipline to Universities (Project 111) from the State Administration of Foreign Experts Affairs (\#B18042) "Crop breeding for disease resistance and genetic improvement".

Conflicts of Interest: The authors declare no conflict of interest.

\section{References}

1. Ahmed, T.; Aslam, M.; Hussan, M.W.; Iqbal, J. Aphids (Schizaphis graminum R.) infectation on different wheat (Triticum aestivum) varieties and their comparative yields. J. Agric. Res. 2015, 53, 209-216.

2. Fu, M.; Xu, M.; Zhou, T.; Wang, D.; Tian, S.; Han, L.; Dong, H.; Zhang, C. Transgenic expression of a functional fragment of harpin protein Hpa1 in wheat induces the phloem-based defence against English grain aphid. J. Exp. Bot. 2014, 65, 1439-1453. [CrossRef] [PubMed]

3. Honek, A.; Martinkova, Z.; Dixon, A.F.G.; Saska, P. Annual predictions of the peak numbers of Sitobion avenae infesting winter wheat. J. Appl. Entomol. 2017, 141, 352-362. [CrossRef]

4. He, Y.; Liu, D.; Dai, D.; Wang, D.; Shi, X. Genetic differentiation and structure of Sitobion avenae (Hemiptera Aphididae) populations from moist, semiarid and arid areas in Northwestern China. J. Econ. Entomol. 2018, 111, 603-611. [CrossRef]

5. Yang, Y.; Yin, Y.; Wang, H.; Yu, Y. lnfluence of honeydew on the wheat yield and nutritive qualities. Chin. Agric. Sci. Bull. 2005, 21, 268-305.

6. Liu, X.F.; Hu, X.S.; Keller, M.A.; Zhao, H.Y.; Wu, Y.F.; Liu, T.X. Tripartite interactions of barley yellow dwarf virus, Sitobion avenae and wheat varieties. PLoS ONE 2014, 9, e106639. [CrossRef]

7. Aziz, M.A.; Ahmad, M.; Nasir, M.F.; Naeem, M. Efficacy of different neem (Azadirachta indica) products in comparison with imidacloprid against English Grain Aphid (Sitobion avenae) on Wheat. Int. J. Agric. Biol. 2013, 15, 279-284.

8. Tétard-Jones, C.; Leifert, C. Plasticity of yield components of winter wheat in response to cereal aphids. NJAS-Wagen. J. Life Sci. 2011, 58, 139-143. [CrossRef]

9. Hu, X.S.; Zhang, Z.F.; Zhu, T.Y.; Song, Y.; Wu, L.J.; Liu, T.X. Maternal effects of the English grain aphids feeding on the wheat varieties with different resistance traits. Sci. Rep. 2018, 8, 7344. [CrossRef]

10. Liu, X.L.; Wang, C.Y.; Wang, Y.J.; Zhang, H.; Ji, W.Q. Screening and evaluation of different wheat cultivars for resistance to Sitobion avenae at seedling and adult-plant stages. Agric. Sci. Technol. 2015, 16, 1686-1692. 
11. Havlíčková, H.; Cvikrová, M.; Eder, J. Phenolic acids in wheat cultivars in relation to plant suitability for and response to cereal aphids. Zeitschrift für Pflanzenkrankheiten und Pflanzenschutz 1996, 10, 535-542.

12. Bahlmann, L.; Govender, P.; Botha, A.M. Leaf epicuticular wax ultrastructure and trichome presence on Russian wheat aphid (Diuraphis noxia) resistant and susceptible leaves. Afr. Entomol. 2003, 11, 59-64.

13. Hao, P.; Liu, C.; Wang, Y.; Chen, R.; Tang, M.; Du, B.; Zhu, L.; He, G. Herbivore-induced callose deposition on the sieve plates of rice: An important mechanism for host resistance. Plant Physiol. 2008, 146, 1810-1820. [CrossRef] [PubMed]

14. Cao, H.H.; Zhang, M.; Zhao, H.; Zhang, Y.; Wang, X.X.; Guo, S.S.; Zhang, Z.F.; Liu, T.X. Deciphering the mechanism of $\beta$-aminobutyric acid-induced resistance in wheat to the grain aphid, Sitobion avenae. PLoS ONE 2014, 9, e91768. [CrossRef] [PubMed]

15. Argandoña, V.H.; Chaman, M.; Cardemil, L.; Munoz, O.; Zuñiga, G.E.; Corcuera, L.J. Ethylene production and peroxidase activity in aphid-infested barley. J. Chem. Ecol. 2001, 27, 53-68. [CrossRef] [PubMed]

16. Reddy, S.K.; Weng, Y.; Rudd, J.C.; Akhunova, A.; Liu, S. Transcriptomics of induced defense responses to greenbug aphid feeding in near isogenic wheat lines. Plant Sci. 2013, 212, 26-36. [CrossRef]

17. Moran, P.J.; Thompson, G.A. Molecular responses to aphid feeding in Arabidopsis in relation to plant defense pathways. Plant Physiol. 2001, 125, 1074-1085. [CrossRef]

18. Losvik, A.; Beste, L.; Glinwood, R.; Ivarson, E.; Stephens, J.; Zhu, L.H.; Jonsson, L. Overexpression and down-regulation of barley lipoxygenase LOX2.2 affects jasmonate-regulated genes and aphid fecundity. Int. J. Mol. Sci. 2017, 18, 2765. [CrossRef]

19. Christensen, S.A.; Nemchenko, A.; Borrego, E.; Murray, I.; Sobhy, I.S.; Bosak, L.; DeBlasio, S.; Erb, M.;

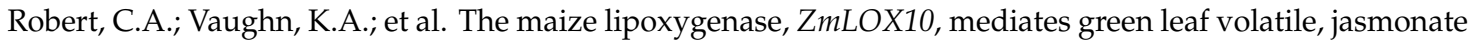
and herbivore-induced plant volatile production for defense against insect attack. Plant J. 2013, 74, 59-73. [CrossRef]

20. Selig, P.; Keough, S.; Nalam, V.J.; Nachappa, P. Jasmonate-dependent plant defenses mediate soybean thrips and soybean aphid performance on soybean. Arthropod-Plant Int. 2016, 10, 273-282. [CrossRef]

21. War, A.R.; Paulraj, M.G.; Sharma, H.C. Induced resistance to Helicoverpa armigera through exogenous application of jasmonic acid and salicylic acid in groundnut, Arachis hypogaea. Pest Manag Sci. 2015, 71, 72-82. [CrossRef] [PubMed]

22. Porta, H.; Rocha-Sosa, M. Plant lipoxygenase. physiological and molecular features. Plant Physiol. 2002, 130, 15-21. [CrossRef] [PubMed]

23. Wang, L.; Allmann, S.; Wu, J.; Baldwin, I.T. Comparisons of LIPOXYGENASE3- and JASMONATERESISTANT4/6-silenced plants reveal that jasmonic acid and jasmonic acid-amino acid conjugates play different roles in herbivore resistance of Nicotiana attenuata. Plant Physiol. 2008, 146, 904-915. [CrossRef] [PubMed]

24. Qin, Q.; Shi, X.; Pei, L.; Gao, X. Induction of phenylalanine ammonia-lyase and lipoxtgenase in cotton seedlings by mechanical wounding and aphid infestation. Prog. Nat. Sci. 2005, 15, 419-423.

25. Nalam, V.J.; Keeretaweep, J.; Shah, J. The green peach aphid, Myzus persicae, acquires a LIPOXYGENASE5derived oxylipin from Arabidopsis thaliana, which promotes colonization of the host plant. Plant Signal. Behav. 2013, 8, e22735. [CrossRef]

26. Escudero-Martinez, C.M.; Morris, J.A.; Hedley, P.E.; Bos, J.I.B. Barley transcriptome analyses upon interaction with different aphid species identify thionins contributing to resistance. Plant Cell Environ. 2017, 40, 2628-2643. [CrossRef]

27. Delp, G.; Gradin, T.; Ahman, I.; Jonsson, L.M.V. Microarray analysis of the interaction between the aphid Rhopalosiphum padi and host plants reveals both differences and similarities between susceptible and partially resistant barley lines. Mol. Genet. Genom. 2009, 281, 233-248. [CrossRef]

28. Smith, C.M.; Liu, X.; Wang, L.J.; Liu, X.; Chen, M.S.; Starkey, S.; Bai, J. Aphid feeding activates expression of a transcriptome of oxylipin-based defense signals in wheat involved in resistance to herbivory. J. Chem. Ecol. 2010, 36, 260-276. [CrossRef]

29. Liu, X.L. Identification of Wheat Germplasm Resistance to English grain aphid (Sitobion avenae F.) and Molecular Mapping and Expression of Resistance Gene; Northwest A\&F University: Yangling, China, 2012.

30. Ni, X.; Quisenberry, S.S.; Markwell, J.; Heng-Moss, T.; Higley, L.; Baxendale, F.; Sarath, G.; Klucas, R. In vitro enzymatic chlorophyll catabolism in wheat elicited by cereal aphid feeding. Entomol. Exp. Appl. 2001, 101, 159-166. [CrossRef] 
31. Sembdner, G. The biochemistry and the physiological and molecular actions of jasmonates. Annu. Rev. Plant Biol. 1993, 44, 569-589. [CrossRef]

32. Bailey, B.A.; Strem, M.D.; Bae, H.; Mayolo, G.A.D. Gene expression in leaves of Theobroma cacao in response to mechanical wounding, ethylene, and/or methyl jasmonate. Plant Sci. 2005, 168, 1247-1258. [CrossRef]

33. Sotherton, N.W.; Emden, H.F.V. Laboratory assessment of resistance to the aphids Sitobion avenae and Metopolophium dirhodum in three Triticum species and two modern wheat cultivars. Ann. Appl. Biol. 2008, 101, 99-107. [CrossRef]

34. Zhai, Y.; Li, P.; Mei, Y.; Chen, M.Y.; Chen, X.C.; Xu, H.; Zhou, X.; Dong, H.S.; Zhang, C.L.; Jiang, W.H. Three MYB genes co-regulate the phloem-based defence against English grain aphid in wheat. J. Exp. Bot. 2017, 68, 4153-4169. [CrossRef] [PubMed]

35. Livak, K.J.; Schmittgen, T.D. Analysis of relative gene expression data using real-time quantitative PCR and the 2(-Delta Delta C(T)) Method. Methods 2001, 25, 402-408. [CrossRef]

36. Sobhy, I.S.; Woodcock, C.M.; Powers, S.J.; Caulfield, J.C.; Pickett, J.A.; Birkett, M.A. Cis-iasmone elicits aphid-induced stress signalling in potatoes. J. Chem. Ecol. 2017, 43, 39-52. [CrossRef]

37. Andreou, A.; Feussner, I. Lipoxygenases-structure and reaction mechanism. Phytochemistry 2009, 70, 504-1510. [CrossRef]

38. Shaban, M.; Ahmed, M.M.; Sun, H.; Ullah, A.; Zhu, L. Genome-wide identification of lipoxygenase gene family in cotton and functional characterization in response to abiotic stresses. BMC Genom. 2018, 19, 599. [CrossRef]

39. Scala, A.; Allmann, S.; Mirabella, R.; Haring, M.A.; Schuurink, R.C. Green leaf volatiles: A plant's multifunctional weapon against herbivores and pathogens. Int. J. Mol. Sci. 2013, 14, 17781-17811. [CrossRef]

40. Bachmann, A.; Hause, B.; Maucher, H.; Garbe, E.; Vörös, K.; Weichert, H.; Wasternack, C.; Feussner, I. Jasmonate-induced lipid peroxidation in barley leaves initiated by distinct 13-LOX forms of chloroplasts. Biol. Chem. 2002, 383, 1645-1657. [CrossRef]

41. Bell, E.; Creelman, R.A.; Mullet, J.E. A chloroplast lipoxygenase is required for wound-induced jasmonic acid accumulation in Arabidopsis. Proc. Natl. Acad. Sci. USA 1995, 92, 8675-8679. [CrossRef]

42. Liu, D.; Cheng, Y.; Gong, M.; Zhao, Q.; Jiang, C.; Cheng, L.; Ren, M.; Wang, Y.; Yang, A. Comparative transcriptome analysis reveals differential gene expression in resistant and susceptible tobacco cultivars in response to infection by cucumber mosaic virus. J. Crop Sci. Engl. Ed. 2019, 3, 307-321.

43. Müller, V.; Amé, M.V.; Carrari, V.; Gieco, J.; Asis, R. Lipoxygenase activation in peanut seed cultivars resistant and susceptible to Aspergillus parasiticus colonization. Phytopatholog 2014, 104, 1340-1348. [CrossRef] [PubMed]

44. Hildebrand, D.F.; Brown, G.C.; Jackson, D.M.; Hamilton-Kemp, T.R. Effects of some leaf-emitted volatile compounds on aphid population increase. J. Chem. Ecol. 1993, 19, 1875-1887. [CrossRef] [PubMed]

45. Jiang, Y.; Ye, J.; Li, S.; Niinemets, Ü. Methyl jasmonate-induced emission of biogenic volatiles is biphasic in cucumber: A high-resolution analysis of dose dependence. J. Exp. Bot. 2017, 68, 4679-4694. [CrossRef] [PubMed]

46. Gális, I.; Gaquerel, E.; Pandey, S.P.; Baldwin, I.T. Molecular mechanisms underlying plant memory in JA-mediated defence responses. Plant Cell Environ. 2009, 32, 617-627. [CrossRef]

47. Glowacz, M.; Bill, M.; Tinyane, P.P.; Sivakumar, D. Maintaining postharvest quality of cold stored 'Hass' avocados by altering the fatty acids content and composition with the use of natural volatile compounds-methyl jasmonate and methyl salicylate. J. Sci. Food Agric. 2017, 97, 5186-5193. [CrossRef]

48. Chen, Z.; Chen, X.; Yan, H.; Li, W.; Cai, R.; Xiang, Y. The lipoxygenase gene family in poplar: Identification, classification, and expression in response to MeJA treatment. PLoS ONE 2015, 10, e0125526. [CrossRef]

(C) 2020 by the authors. Licensee MDPI, Basel, Switzerland. This article is an open access article distributed under the terms and conditions of the Creative Commons Attribution (CC BY) license (http://creativecommons.org/licenses/by/4.0/). 